\title{
El documento administrativo electrónico defectuoso: ¿una nueva categoría?
}

\author{
Antonio David Berning Prieto (España) \\ Universidad Pablo de Olavide \\ aberning@upo.es
}

\begin{abstract}
NOTA BIOGRÁFICA
Licenciado en Derecho por la Universidad Pablo de Olavide (2010), Máster Universitario en Derechos Fundamentales por la Universidad Nacional de Educación a Distancia (2012), Máster Universitario en Derecho de las Nuevas Tecnologías por la Universidad Pablo de Olavide (2014), beneficiario de FPU en el periodo 2014-2018, actualmente Colaborador en el Área de Derecho Administrativo (Departamento de Derecho Público) de la Facultad de Derecho de la Universidad Pablo de Olavide, centra sus líneas de investigación fundamentalmente en administración electrónica.
\end{abstract}

\section{RESUMEN}

El presente trabajo pretende, a la vista de la novedosa regulación jurídico-administrativa de los requisitos documentales en soporte electrónico, efectuar una propuesta para distinguir conceptualmente aquellos vicios que pueden afectar a los documentos administrativos en general de los que afectan a los actos administrativos en particular, calificando de forma distinta unos y otros con el fin de concluir determinando las consecuencias que en uno u otro caso debe conllevar la apreciación de tales vicios. De este modo se consigue dar un tratamiento adecuado a esta novedosa situación que puede presentarse y proporcionar seguridad jurídica en la actuación administrativa.

\section{PALABRAS CLAVE}

Documento; acto; electrónico; invalidez; defectuoso; perfecto.

\begin{abstract}
In view of the new administrative law regulation of the requirements imposed on administrative documents in electronic support, the present work aims at making a proposal to distinguish conceptually those vices that may affect the administrative documents in general from those that affect administrative resolutions in particular, interpreting them differently in order to conclude determining the consequences that in each case should lead to the appreciation of such vices. We succeed thus in giving a suitable treatment to this new situation that can arise in practice, and to provide legal certainty in the administrative activity.
\end{abstract}

\section{KEYWORDS}

Document; resolution; electronic; invalidity; defective; perfect.

\section{SUMARIO}

I. EL CONCEPTO DE DOCUMENTO. II. EL DOCUMENTO ADMINISTRATIVO ELECTRÓNICO. III. EL DOCUMENTO ADMINISTRATIVO ELECTRÓNICO DEFECTUOSO. IV. LAS COPIAS ELECTRÓNICAS DEFECTUOSAS. V. LOS VICIOS DOCUMENTALES DEL ACTO ADMINISTRATIVO. VI. CONSECUENCIAS DEL INCUMPLIMIENTO DE LOS REQUISITOS DE CARÁCTER DOCUMENTAL DEL ARTÍCULO 26.2 LPAC. VII. CONCLUSIONES. 


\section{EL CONCEPTO DE DOCUMENTO}

El documento ha sido, tradicionalmente, el medio a través del cual el hombre ha plasmado la información susceptible de conservación, precisamente para conseguir el almacenamiento de la misma y hacerla perdurar en el tiempo y, como indica (VALCÁRCEL FERNÁNDEZ, 2011: 542), ha servido históricamente a la humanidad para registrar datos, originalmente como inventario de bienes entre otras finalidades, evolucionando especialmente en las épocas griega y romana hasta erigirse en el elemento base de las relaciones jurídicas. El concepto de documento es relevante, como apunta (HEREDIA HERRERA, 2007: 14), ya que «los nombres de las cosas son esenciales para el conocimiento de estas», por lo que debe en primer lugar identificarse qué debe entenderse por «documento».

Acudiendo en primer lugar al concepto incluido en el Diccionario de la Real Academia Española ${ }^{1}$, según el cual el término proviene del latín documentum, se puede definir, empleando una de las acepciones más acordes al fin del presente trabajo, como «escrito en que constan datos fidedignos o susceptibles de ser empleados como tales para probar algo». La doctrina también ha proporcionado otras conceptualizaciones del término y, en este sentido, (CRUZ MUNDET, 2009: 64) lo identifica con el soporte de actividades internas, predecibles y reguladas, como lo son la información que contienen, y otros autores como (SENDÍN GARCÍA, 2009: 55-56), con la conjunción material de tres elementos: el soporte, la información o mensaje que transmite, y la fijación de dicha información en el soporte.

Por su parte, (GAETE GONZÁLEZ, 2009:121) establece que los elementos del documento son:

1. Elemento corporal: lo identifica el autor como el cuerpo del documento, que comprende a su vez dos aspectos:

a) Corpus, como el documento físicamente considerado. Debe tenerse en cuenta que la concepción tradicionalmente admitida de "documento físico» debe entenderse superada en la actualidad, puesto que el documento «electrónico» está formado (codificado) por bits, que son una combinación de dígitos -ceros y unos $(0,1)$-, que requieren la intervención de un sistema informático para descodificarlos y transformarlos a un lenguaje inteligible por humanos.

b) Grafía, como medio de expresión que se incorpora al cuerpo del documento por parte de su autor, integrado a su vez por dos aspectos: el estático, como medio de producción de las formas, y el dinámico, como enlace entre la mente del autor y el documento, que se transmite posteriormente a los destinatarios.

3. Elemento intelectual: comprende tanto la conciencia o intención expresiva al momento de elaborar el documento, como la expresividad explicativa del autor dirigida al destinatario. Este elemento comprende a su vez aspectos como la expresión del pensamiento del sujeto o su significación jurídica, y la recognoscibilidad, esto es, la existencia de hechos jurídicos y elementos formales, entre otros, resultando relevante de estos últimos la fijación en el documento de la información. El soporte electrónico es, en este sentido, un nuevo medio en virtud del cual se genera un documento a partir de la plasmación, a través de programas y sistemas informáticos, de la información que contiene.

Conforme con lo expuesto, cualquier documento, público o privado, puede encontrarse en soporte físico (impreso en papel) o electrónico (en una unidad de almacenamiento), sirviendo en ambos casos de forma idéntica a su finalidad.

\section{EL DOCUMENTO ADMINISTRATIVO ELECTRÓNICO}

Como es sabido, la entrada en vigor ${ }^{2}$ de la Ley 39/2015, de 1 de octubre, del Procedimiento Administrativo Común de las Administraciones Públicas (LPAC, en adelante), ha supuesto un cambio de paradigma en

Se puede consultar la versión online del Diccionario de la Real Academia Española a través de la dirección web: http://dle.rae. es/?id=E4EdgX1 (última consulta efectuada el 31-10-2018).

2 Debe tenerse en cuenta que, si bien la Disposición final séptima establecía su entrada en vigor al año de su publicación en el Boletín Oficial del Estado (BOE), dicha disposición ha sido objeto de modificación a tenor del Real Decreto-ley 11/2018, de 31 de agosto, de transposición de directivas en materia de protección de los compromisos por pensiones con los trabajadores, prevención del blanqueo de capitales y requisitos de entrada y residencia de nacionales de países terceros y por el que se modifica la Ley 39/2015, de 1 de octubre, del Procedimiento Administrativo Común de las Administraciones Públicas, en el sentido de que las previsiones relativas 
el funcionamiento ad intra y ad extra de las Administraciones Públicas, ya que de la potestativa tramitación electrónica de procedimientos se ha pasado al otro extremo, esto es, a su preceptividad, llegando incluso a obligarse a determinados sujetos y colectivos a relacionarse electrónicamente con la Administración, al presumírseles dotados de medios y conocimientos suficientes para ello.

Por ende, la Administración Pública tiene dos cometidos claramente diferenciados pero interrelacionados entre sí e incluso dependientes el uno del otro: transformar su gestión interna a una íntegra tramitación electrónica, y habilitar canales de comunicación y relación electrónica con los ciudadanos para permitir la bidireccionalidad comunicativa y procedimental, plasmando todos los actos, documentos y actuaciones en soporte electrónico. Y se dice «todos» porque, a pesar de que la LPAC no establezca la obligación de relacionarse electrónicamente con la Administración a todas las personas físicas (sí lo hace respecto a las personas jurídicas), a la Administración sí se le obliga a tramitar electrónicamente todos los procedimientos, estableciéndose la diferencia en la notificación, que en el caso de los no obligados a relacionarse electrónicamente con la Administración se efectuará tanto en papel como electrónicamente (a través de la sede electrónica), mientras que a los obligados se les efectuará siempre a través de medios electrónicos (artículo 42.1 LPAC).

Ello conlleva la necesidad del legislador de establecer determinados requisitos que deben cumplir las diversas caras del prisma, las cuales, en conjunción, dan forma a la administración electrónica, una de las cuales es la documentación administrativa, ahora generada en su totalidad en soporte electrónico.

En la vigente normativa reguladora del procedimiento administrativo se contienen las exigencias impuestas a todo documento administrativo, concretándose en el artículo 26.2 LPAC, al disponerse que:

«Para ser considerados válidos, los documentos electrónicos administrativos deberán:

a) Contener información de cualquier naturaleza archivada en un soporte electrónico según un formato determinado susceptible de identificación y tratamiento diferenciado.

b) Disponer de los datos de identificación que permitan su individualización, sin perjuicio de su posible incorporación a un expediente electrónico.

c) Incorporar una referencia temporal del momento en que han sido emitidos.

d) Incorporar los metadatos mínimos exigidos.

e) Incorporar las firmas electrónicas que correspondan de acuerdo con lo previsto en la normativa aplicable.

Se considerarán válidos los documentos electrónicos que, cumpliendo estos requisitos, sean trasladados a un tercero a través de medios electrónicos».

Como puede apreciarse, la normativa reguladora del procedimiento administrativo ha impuesto a las Administraciones Públicas la obligación de observar determinadas prescripciones a la hora de generar documentos administrativos, como condicionantes para lo que el legislador ha venido a denominar de «validez», a saber:

- Conservación de información en un soporte electrónico conforme a un formato determinado que pueda tratarse e identificarse diferenciadamente.

- Incorporación de datos de identificación que lo diferencien del resto.

- Inclusión de referencia temporal, sin que se exija por tanto la necesidad de sello electrónico de tiempo o time stamping.

- Asignación de los metadatos mínimos exigidos.

- Incrustación de la firma electrónica que proceda.

Se exigen, por tanto, una serie de requisitos para entender válidamente generado un documento administrativo en soporte electrónico, algunos de ellos de contenido evidente, como que el documento contenga algún tipo de información, siendo por tanto el objeto material del documento administrativo, según (SANZ LARRUGA, 2017: 938), cualquier información o actuación procedente de una Administración identificada y diferenciada, pudiendo un mismo documento formar parte de dos expedientes administrativos electrónicos distintos (artículo 70.3 LPAC), por ejemplo, gracias a que la Ley exige que disponga de datos de identifica-

al registro electrónico de apoderamientos, registro electrónico, registro de empleados públicos habilitados, punto de acceso general electrónico de la Administración y archivo único electrónico producirán efectos con posterioridad a lo inicialmente previsto, concretamente a partir del 2 de octubre de 2020. 
ción que lo hagan único e individualizado, y otros menos evidentes para un ciudadano común pero necesarios, como la incorporación de los metadatos, (al menos los mínimos obligatorios previstos por las normas técnicas de interoperabilidad, en especial la Norma Técnica de Interoperabilidad de Documento Electrónico, aprobada mediante Resolución de la Secretaría de Estado para la Función Pública de 19 de julio de 2011) gracias a los cuales se puede, por ejemplo, distinguir un documento original de su copia (ya que un documento electrónico puede tener infinitas instancias de sí mismo, dado su soporte), y se podrá igualmente hacer constar el órgano del que proviene, así como el expediente original en el que se encontraba.

Alguno de estos requisitos tiene una relevancia capital, como la incorporación de la firma electrónica, la cual proporciona al documento características fundamentales tan necesarias como la integridad, autenticidad y autoría del mismo, garantizando de este modo, entre otros, que el documento no ha sido modificado desde el momento en que se generó, mientras que otros requisitos, a pesar de su importancia, no han sido objeto, a mi juicio, de una adecuada regulación normativa, como la mera exigencia de referencia temporal a todo documento administrativo, sea cuál sea la naturaleza de éste. A este respecto, lo deseable hubiese sido la previsión de algunos supuestos en que resultase preceptiva la inclusión de un sello electrónico de tiempo, a efectos de incrementar sus facultades probatorias, en aquellos casos en que la relevancia del mismo o la posible afectación de derechos de terceros así lo exigiese ya que, como es bien sabido, se trata de un certificado electrónico en el que una autoridad (tercero) debidamente acreditada proporciona una suerte de fe digital del momento exacto en el que ha sido incorporado dicho certificado al documento.

Por último, debe tenerse en cuenta que las normas técnicas de interoperabilidad «derivadas» del Esquema Nacional de Interoperabilidad, aprobado por Real Decreto 4/2010, de 8 de enero, por el que se regula el Esquema Nacional de Interoperabilidad en el ámbito de la Administración Electrónica, tales como las de Expediente Electrónico, Digitalización de Documentos, Catálogo de Estándares, de Gestión de Documentos Electrónicos o de Modelos de Datos, entre otras, aprobadas mediante sendas resoluciones de la Secretaría de Estado para la Función Pública, establecen requisitos adicionales específicamente desarrollados para cada ámbito concreto de aplicación que deben observarse en aras a una correcta generación de documentos en soporte electrónico.

\section{EL DOCUMENTO ADMINISTRATIVO ELECTRÓNICO DEFECTUOSO}

A pesar de que el legislador ha considerado procedente el establecimiento de requisitos de «validez» de los documentos administrativos cuando son generados en soporte electrónico en el anteriormente citado artículo 26.2 LPAC, lo cierto es que los términos «validez» e «invalidez» son calificaciones que deben reservarse, bajo mi punto de vista, para los actos administrativos como tipología específica documental, y que identifican una concreta institución jurídica que no ha sido concebida para cualquier tipología documental.

$Y$ es que lo que, en definitiva, aquí se defiende, es que los vicios de que puede adolecer un acto administrativo no deben confundirse con aquellos que pueden afectar a cualquier otro documento generado por la Administración y, por tanto, su calificación no puede ser la misma.

La (in)validez es una institución jurídica concebida para una concreta categoría documental: el acto administrativo, y a éste debe reservarse, debiendo por tanto establecerse una denominación distinta para aquellos casos en los que cualquier otro documento administrativo en soporte electrónico, distinto del acto, incumple cualquier requisito de los establecidos en el artículo 26.2 LPAC con carácter general.

En este sentido, el que suscribe considera que el documento administrativo electrónico debe calificarse, en caso de verificar todos los requisitos del precitado artículo 26.2 LPAC, como «perfecto», mientras que, en caso contrario, sería «defectuoso».

Para la elección del término, tras consultar el Diccionario de la Real Academia Española ${ }^{3}$, se ha advertido que el adjetivo "defectuoso», proveniente del latín defectus, en una de sus acepciones es «imperfecto», por lo que, tomando así mismo el antónimo de este último, se estima que los documentos administrativos en general deben ser denominados «defectuosos» o «perfectos», en función de si adolecen o no de algún vicio, respectivamente.

Así, partiendo de la base de que el incumplimiento de determinados requisitos del artículo 26.2 LPAC no es baladí, y debe contemplarse una calificación específica para el documento viciado que debe diferir

3 Puede consultarse la versión online del mismo en la dirección web: $h$ ttp://dle.rae.es/?id=C1jRL3D (última consulta efectuada el 31-10-2018). 
de la específicamente concebida para los actos administrativos (invalidez), debe ponerse de manifiesto que la denominación propuesta no resulta totalmente abstracta y sin precedentes, sino que la misma ya está contemplada en el ordenamiento jurídico en materia de notificaciones, las cuales se califican por la doctrina como «defectuosas» cuando incumplen determinadas prescripciones normativas (MARTÍN DELGADO, 2017: 2.151; GAMERO CASADO y FERNÁNDEZ RAMOS, 2017: 561-562 ).

Dicho incumplimiento no provoca, como es unánimemente admitido por la doctrina, la invalidez del acto, sino que se califica la notificación como «defectuosa», y se le priva al acto de su facultad de desplegar los efectos que le son inherentes, esto es, impide que el mismo sea eficaz, sin que en ningún momento resulte afectada su validez.

Por otra parte, cuando el artículo 26.2 LPAC enumera los requisitos de "validez», se estima igualmente que alguna consecuencia deberá conllevar su incumplimiento, a pesar de que no haya sido objeto de regulación expresa por parte del legislador.

Si fuese un acto administrativo, para determinar dicha consecuencia lo primero que se requiere sería su calificación como nulo o anulable, para, acto seguido, prever determinadas consecuencias inherentes a dicha calificación; de tratarse de cualquier otro documento administrativo, al no existir categorías como éstas ni estimarse en este caso necesario, se considera adecuado englobarlas todas en la de documentos «defectuosos», lo cual igualmente deberá llevar aparejadas determinadas consecuencias, como se verá.

También podrían aparecer defectos de escasa gravedad, al igual que los actos administrativos pueden presentar meras irregularidades no invalidantes, que podrían ocasionar la generación de un documento administrativo electrónico meramente «irregular», no perfecto ni defectuoso, que la Administración podría corregir de oficio o a instancia del interesado en cualquier momento, de la misma forma que con aquéllos.

Del mismo modo, si bien es cierto que merece ser criticada la decisión del legislador de ubicar sistemáticamente los requisitos de los documentos administrativos electrónicos en sede de la actividad de las Administraciones Públicas, concretamente en las normas generales de actuación (Capítulo I del Título II de la LPAC), en lugar de ubicar su contenido en un Título y/o Capítulo independiente dedicado a la regulación de los documentos administrativos y sus copias, lo cual hubiese resultado en una regulación uniforme y con mejor sistemática tanto de sus requisitos y exigencias como de las consecuencias o efectos de su inobservancia, no lo es menos que dicha decisión parece apoyar la propuesta aquí presentada de reconocer el documento electrónico defectuoso como un nuevo concepto dogmático, distinto del acto administrativo inválido, e independiente de las causas o vicios de nulidad y anulabilidad actualmente reconocidas pero que, a su vez, de afectar a actos administrativos, sí podría dar lugar a estos vicios, como se verá.

\section{LAS COPIAS ELECTRÓNICAS DEFECTUOSAS}

Al igual que los documentos administrativos en soporte electrónico, el régimen de realización de copias ha sido objeto de una novedosa regulación llevada a cabo en el artículo 27 LPAC, distinguiendo hasta cinco tipologías. Además de los requisitos específicos regulados en este precepto, debe tenerse en cuenta que también se han establecido requisitos adicionales específicos en la Norma Técnica de Interoperabilidad de Procedimientos de copiado auténtico y conversión entre documentos electrónicos así como en la de Digitalización de Documentos, aprobadas ambas mediante Resolución de 19 de julio de 2011, de la Secretaría de Estado para la Función Pública.

De la misma forma que los documentos administrativos en soporte electrónico con carácter general, la copia electrónica es, en realidad, un documento administrativo que cumple determinadas características, las cuales le confieren, en caso de verificarse, la calificación y cualificación de auténtica, puesto que adquieren de esta forma un valor probatorio reforzado, al haber sido expedidas por el funcionario público habilitado para la expedición de copias auténticas, cuya intervención proporciona una especie de fe pública en relación con la identidad entre el original y la copia.

Pues bien, en caso de incumplir las copias realizadas por las Administraciones Públicas algún requisito de los específicamente previstos para cada una de las tipologías reguladas en la Ley, la misma no podría considerarse auténtica y, por ende, quedará desprovista de su valor probatorio adicional, debiendo otorgársele similar valor que a la copia privada.

Por su parte, en caso de incumplir la copia cualquiera de los requisitos documentales previstos en el artículo 26.2 LPAC el cual, como se ha visto, resulta de aplicación a todo documento, también al «documentocopia», la misma deberá calificarse como defectuosa, mientras que, si reúne todos aquellos requisitos, la 
copia será perfecta ya que, al tratarse, en definitiva, de un documento administrativo electrónico, no de una institución en sí misma como sucede con el acto administrativo, se estima adecuado adoptar la terminología general propuesta para el mismo.

También, como en el caso de los documentos administrativos en soporte electrónico en general, pueden presentarse vicios de menor calado que no provoquen que la copia devenga defectuosa, sino que deben considerarse igualmente meras irregularidades que dan lugar a una copia meramente irregular, debiendo en este caso procederse a su subsanación por parte de la Administración, de oficio o a instancia de interesado.

Evidentemente, la calificación de una copia como defectuosa o meramente irregular dependerá, en definitiva, de la gravedad del vicio de que adolezca, en función del requisito incumplido y la función que el mismo ostenta en relación con el documento-copia.

Aunque el examen pormenorizado de todos los supuestos que pueden darse en la práctica en relación con tales requisitos excedería de los límites propios del presente trabajo ${ }^{4}$, baste ejemplificar al respecto que no puede asimilarse, por ejemplo, la ausencia de firma electrónica, que no es sino un certificado electrónico emitido por un prestador de servicios de confianza, a la ausencia del metadato que identifica al documento como «original» o "copia», puesto que la primera garantiza la integridad, autenticidad y autoría del documento-copia, mientras que el segundo es un mero descriptor del tipo documental concreto de que se trata, por lo que las consecuencias en cada caso no pueden ser las mismas.

Otro supuesto que podría darse sería la digitalización de un original con una resolución distinta a la mínima exigida en la Norma Técnica de Interoperabilidad de Digitalización de Documentos, caso en el que la misma sería defectuosa si impidiese visualizar su contenido correctamente, mientras que si fuese posible el acceso al contenido constituiría una mera irregularidad susceptible de subsanación y la copia no devendría defectuosa.

\section{LOS VICIOS DOCUMENTALES DEL ACTO ADMINISTRATIVO}

El acto administrativo, entendido éste, siguiendo la definición de ZANOBINI adaptada por (GARCÍA DE ENTERRÍA y FERNÁNDEZ RODRÍGUEZ, 2013: 591), como toda declaración de voluntad, de juicio, de conocimiento o deseo realizada por la Administración en ejercicio de una potestad administrativa distinta de la reglamentaria, en la actualidad aparece con la especialidad de ser dictado en soporte electrónico perteneciendo, por tanto, a la categoría genérica del documento administrativo electrónico, toda vez que se trata, en definitiva, de un tipo específico documental.

Por ende, todo acto administrativo dictado en soporte electrónico, como documento administrativo electrónico, deberá cumplir no sólo los requisitos tradicionales en relación con sus elementos subjetivo, objetivo, causal y formal, sino que además deberá cumplir los requisitos que se exigen, con carácter general, a todo documento administrativo electrónico en el artículo 26.2 LPAC.

Por tanto, al encontrarnos ante un acto administrativo en soporte electrónico, no puede obviarse que el mismo podría sufrir alteraciones debidas no únicamente a su condición de acto, sino también a la de documento, y que ello podrá conllevar también especialidades que deben ser tenidas en cuenta, a pesar de no haber reparado en ello el legislador.

En este sentido, se ha obviado total y absolutamente en la regulación de la invalidez de la LPAC toda referencia al incumplimiento del artículo 26.2 incluso para el caso de que afectase no ya a los documentos administrativos, sino a los propios actos administrativos, lo cual resulta del todo reprochable, en tanto en cuanto que se deja al arbitrio de la interpretación que efectúen los distintos órganos, administrativos y judiciales, en relación con las concretas consecuencias que su ausencia o incorrección implican, provocando así una evidente inseguridad jurídica a los administrados e incluso a las Administraciones Públicas, difícil de salvar en la práctica.

En el caso del acto administrativo, sin perjuicio de la crítica que merece así mismo la prácticamente idéntica regulación de la institución por parte del legislador respecto a la regulación previa de la Ley 30/1992, de 26 de noviembre, de Régimen Jurídico de las Administraciones Públicas y del Procedimiento Administrativo Común -a salvo la impericia consistente en la calificación como nula de las infracciones reglamentarias

4 Para el examen en profundidad de tales cuestiones procede remitir a la tesis doctoral del autor: BERNING PRIETO, A. D. (en prensa): Validez e invalidez de actos y documentos administrativos en soporte electrónico, Thomson Reuters Aranzadi: Cizur Menor (Navarra). 
del artículo 37.2 LPAC, probablemente originada por un malogrado intento de incorporar en la legislación procedimental determinadas previsiones específicas del artículo 23.4 de la Ley 50/1997, de 27 de noviembre, del Gobierno, el cual disponía que «son nulas las resoluciones administrativas que vulneren lo establecido en un reglamento, aunque hayan sido dictadas por órganos de igual o superior jerarquía que el que lo haya aprobado», como ha tenido ocasión de apuntar (GALÁN VIOQUE, 2017: 2.031-2.032)-, lo cierto es que el incumplimiento de los requisitos documentales puede afectar a su validez, provocando que un acto administrativo dictado cumpliendo todos los requisitos propios de éste, adolezca de nulidad o anulabilidad por no observar, al ser generado en soporte electrónico, alguno de los requisitos establecidos en el artículo 26.2 LPAC.

Por tanto, en primer lugar, hay que tener en cuenta que, para calificar un acto inválido como nulo o anulable sobre la base de los defectos documentales, es preciso integrarlo en los tradicionales vicios de invalidez relacionados en los artículos 47 y 48 LPAC, esto es, deben subsumirse en éstos los relativos a su carácter de documento.

$\mathrm{Y}$, en segundo lugar, en función de su gravedad, cada supuesto habrá de reconducirse a una u otra categoría (nulidad o anulabilidad) para conferirles el correspondiente régimen jurídico en cuanto a las consecuencias o efectos de la declaración de invalidez, que es precisamente lo que debió llevar a cabo el legislador al regular los requisitos de todo documento administrativo, ya que la ausencia de regulación ha dado lugar a una laguna jurídica difícil de salvar garantizando la seguridad jurídica si no existen unas claras consecuencias o efectos para el caso de su incumplimiento, con distinción en función de si afecta a un documento o a un acto administrativo, como se viene defendiendo.

Así, en relación con los actos administrativos que adolecen de defectos documentales, debe partirse de la dicción del artículo 48.2 LPAC, en virtud del cual el defecto de forma sólo determina la anulabilidad cuando el acto carezca de los requisitos formales indispensables para alcanzar su fin o dé lugar a indefensión de los interesados ya que, en principio, fuera de estos supuestos, el acto adolecería de una mera irregularidad no invalidante.

Si bien podría defenderse que cualquier defecto vinculado a los requisitos del artículo 26.2 LPAC estaría siempre relacionado con la forma del documento y, por ende, podría incardinarse en los conocidos vicios de forma, debe examinarse convenientemente cada uno de ellos para determinar si realmente hay un vicio de carácter material, superando el estrictamente formal, al incumplirse alguno de ellos, o si, aun siendo de carácter formal, el vicio realmente impide al acto administrativo final alcanzar su fin o provoca indefensión a los interesados y, por ende, adolece de anulabilidad, superando así la mera irregularidad no invalidante.

Para no resultar excesivamente abstracto, conviene en cualquier caso exponer algún ejemplo, como sería la utilización de un formato determinado para dictar el acto administrativo ex artículo 26.2.a) LPAC, distinto a los previstos en las diversas normas técnicas. En este supuesto, en principio, dicho vicio documental no comportaría más que una mera irregularidad no invalidante; no obstante, sería un vicio de forma que daría lugar a la anulabilidad del acto en el caso de que el mismo careciese de los requisitos esenciales para alcanzar su fin o produzca indefensión a los interesados. Igualmente, en el caso ya comentado anteriormente de ausencia de firma electrónica, el acto devendría inválido (anulable) por resultar imposible garantizar la integridad, autenticidad y autoría del mismo, a cuyo fin responde la incorporación de tales certificados electrónicos.

\section{CONSECUENCIAS DEL INCUMPLIMIENTO DE LOS REQUISITOS DE CARÁCTER DOCUMENTAL DEL ARTÍCULO 26.2 LPAC}

En relación con las consecuencias o efectos de la calificación de un acto administrativo como «inválido» o un documento administrativo como «defectuoso» sobre la base de defectos documentales por incumplimiento del artículo 26.2 LPAC, al encontrarse ambos en soporte electrónico, se parte de la premisa de que tales vicios exceden de las meras irregularidades, las cuales no darían lugar a ninguna consecuencia jurídica más allá de la obligación de corrección o subsanación por parte de la Administración en cualquier momento.

Así, en primer lugar, debe considerarse que, de tratarse de un acto administrativo, más allá de la consideración de los vicios meramente formales que constituyen irregularidades no invalidantes (salvo que causen indefensión o impidan al acto alcanzar su fin ex artículo 48.2 LPAC), cuando los vicios están relacionados con los requisitos de todo documento administrativo en soporte electrónico del artículo 26.2 LPAC 
dará lugar a alguna de las conocidas graduaciones de la invalidez, esto es, nulidad absoluta o anulabilidad, en función de la gravedad del vicio, cuyo examen detenido excedería los límites del presente trabajo ${ }^{5}$, al tratarse de múltiples supuestos los que podrían darse.

En cuanto a los efectos o consecuencias propias de la invalidez, deben distinguirse, con (CANO CAMPOS, 2017: 1.909), entre los efectos de carácter material y los aspectos temporales derivados de dicha invalidez.

Aunque el Derecho establece determinados requisitos de validez y, en caso de incumplimiento, el acto viciado no debería desplegar efectos como principal y directa consecuencia del mero hecho de estar viciado, la presunción de validez de los actos y su ejecutividad como privilegio otorgado por el ordenamiento jurídico a la Administración hacen que, hasta que no se declare por el órgano competente en cada caso, ya administrativo, ya judicial, dicha invalidez no opera materialmente y, por ende, el acto sigue siendo válido a efectos prácticos y desplegando los efectos materiales que le son inherentes, motivo por el cual resulta patente que las tradicionales propiedades que caracterizan y vienen distinguiéndose para ambas categorías de la invalidez (tales como que la nulidad absoluta opera ipso iure o que su apreciación es meramente declarativa, en contraposición a la anulabilidad) no tienen virtualidad práctica real, siendo en realidad una rémora teórica del origen de la institución, que se remonta al Derecho Romano en el ámbito de las relaciones privadas, posteriormente adoptado por el Derecho Público.

Por tanto, una vez declarada su invalidez (y no antes), el acto deja de ser eficaz y, por ende, no podrá continuar desplegando efectos. No obstante, se plantea el interrogante de qué ocurre exactamente con los efectos que ya ha desplegado durante su previa ejecutividad y ejecutoriedad inmediatas, producto de la presunción de su validez, esto es, los aspectos temporales a que se refería CANO CAMPOS, puesto que, lo conveniente y deseado por el Derecho, es la eliminación de todo efecto ilegalmente producido, sin perjuicio de la vocación de permanencia de los actos.

Esta vocación se materializa en las técnicas de conservación y sanación de los actos viciados cuando concurren determinadas circunstancias, así como los límites a la extensión de la nulidad o anulabilidad de los actos (artículos 49 y ss. LPAC) u operan los límites generales del artículo 110 LPAC en materia de revisión, así como principios como el de buena fe, proscripción del enriquecimiento injusto o el de confianza legítima, lo cuales permiten en determinados supuestos la conservación del acto ilícito.

En segundo lugar, de tratarse de un documento administrativo en soporte electrónico, la cuestión es que, como se ha defendido, no es posible aplicar al mismo las categorías propias de la invalidez de los actos administrativos y, por ende, de incurrir en alguno de los vicios examinados, dará lugar a un documento defectuoso o meramente irregular. En el primer caso, siempre y cuando los vicios de que adolezca ostenten cierta relevancia o gravedad, puesto que las irregularidades se reservan a aquellos incumplimientos del ordenamiento jurídico que no adquieran especial relevancia, no afecten a derechos de terceros ni les produzcan indefensión.

Por tanto, al tratarse de vicios de cierta gravedad, y dan por tanto lugar a la calificación del documento administrativo electrónico como defectuoso o del acto como inválido, las consecuencias también deberán ser proporcionales a dicha gravedad, sin que se estime adecuado mantener los efectos materiales que un documento perfecto o un acto válido desplegarían en la realidad jurídica de haber sido generados con pulcra observancia de los requisitos del artículo 26.2 LPAC.

En el caso del documento administrativo electrónico defectuoso, se considera que la principal consecuencia o efecto de dicha calificación debe ser la ineficacia del documento, de forma que el mismo no pueda desplegar los efectos que le son inherentes.

Dado que el documento electrónico defectuoso no debe desplegar efectos, la actuación necesaria por parte de la Administración autora del mismo será la subsanación de los defectos de que adolezca el mismo, para así poder continuar, en su caso, con la tramitación del procedimiento administrativo en el que se ha generado aquél ya que, de tratarse, por ejemplo, de informes preceptivos, toda decisión posterior podría devenir viciada también en caso de no procederse a la debida subsanación, con los consiguientes perjuicios para el interés público que dicha situación podría originar. Por tanto, una actuación responsable por parte de la Administración resulta en este punto, subsanando el defecto, resulta totalmente exigible e imprescindible.

${ }^{5}$ Dicha cuestión ha sido también ampliamente desarrollada en la tesis doctoral del autor, para cuya referencia vid. supra nota al pie anterior. 


\section{CONCLUSIONES}

Dada la novedosa regulación de los requisitos de los documentos administrativos electrónicos y la ausencia de una regulación de las consecuencias en caso de incumplimiento distinta a la regulación de los vicios de los actos administrativos, se propone la distinción de ambas categorías: la del documento electrónico defectuoso por incumplir alguno de los requisitos del artículo 26.2 LPAC, de un lado, y la del acto administrativo inválido por adolecer de algún vicio de nulidad o anulabilidad conforme a los artículos 47 y 48 LPAC, respectivamente, de otro.

En este sentido, también las copias electrónicas, como documentos administrativos que, en definitiva, son, también pueden ser defectuosas de incumplir alguno de los requisitos de carácter documental y, en determinados supuestos, ante el incumplimiento de requisitos tales como la realización por personal funcionario habilitado, perderán su carácter de auténtica, pasando a tener el valor de meras copias privadas.

Por su parte, en el caso de los actos administrativos, a los tradicionales vicios que provocan su invalidez deberán adicionarse los regulados con carácter general para los documentos electrónicos en el artículo 26.2 LPAC y, en función de la gravedad de los mismos o la posible afectación de derechos de terceros, graduarlos, siendo no obstante la anulabilidad la regla general, a tenor del artículo 48.1 LPAC.

También debe reconocerse, en estos casos, la posible existencia de meras irregularidades, que en el caso del documento electrónico o las copias no los convierten en defectuosas, ni en el caso del acto administrativo provocan su invalidez, sino su carácter «irregular», pudiendo rectificarse en cualquier momento ex artículo 109.2 LPAC, previsto actualmente sólo para los actos administrativos, por lo que sería también deseable que se hiciese alguna previsión expresa para los documentos administrativos y las copias, en aras a una mayor seguridad jurídica.

Al respecto, se propone también una regulación diferenciada de las meras irregularidades no invalidantes que afectan a los actos administrativos, y aquellas irregularidades que afectan al documento administrativo en general y, por tanto, no lo hacen defectuoso, sino irregular. Por supuesto, al tratarse el acto administrativo de un documento administrativo, los motivos que provoquen la irregularidad del documento administrativo podrían afectar también al acto; sin embargo, los motivos afectantes al acto administrativo únicamente serían de aplicación a éste, dada su especificidad y naturaleza especial dentro del concepto genérico de documento administrativo (dicho esto con la salvedad de los actos administrativos no documentados, como órdenes orales o acústicas), y su regulación especial en el artículo 109.2 LPAC.

En el escenario electrónico ante el que nos encontramos, mantener la tradicional regulación de la invalidez de los artículos 47 y 48 LPAC, así como de las meras irregularidades no invalidantes del artículo 109.2 LPAC, propias hasta ahora, como se ha dicho, del acto administrativo, para su aplicación, por analogía, a los documentos administrativos electrónicos en general, no es lo más coherente de acuerdo con una buena técnica legislativa, más aún cuando la LPAC ha regulado, en su artículo 26.2, requisitos específicos aplicables a éstos.

Una conveniente regulación diferenciada no sólo redundará en beneficio del interés público, sino que beneficiará a las Administraciones Públicas, al poder actuar con uniformidad ante las diversas situaciones que en la práctica puedan darse, y proporcionará al mismo tiempo a los administrados la debida seguridad jurídica del que el ordenamiento jurídico-administrativo y aquéllas son garantes.

\section{REFERENCIAS BIBLIOGRÁFICAS}

CANO CAMPOS, T. (2017): "Validez e invalidez de los actos administrativos: teoría general”, en GAMERO CASADO, E. (dir.): Tratado de Procedimiento Administrativo Común y Régimen Jurídico Básico del Sector Público, Tomo II, Valencia: Tirant Lo Blanch.

CRUZ MUNDET, J. R. (2009): Qué es un archivero, Asturias: Trea.

GAETE GONZÁLEZ, E. A. (2009): Instrumento público electrónico, Barcelona: Bosch.

GALÁN VIOQUE, R. (2017): "Anulabilidad e irregularidades no invalidantes", en GAMERO CASADO, E. (dir.): Tratado de Procedimiento Administrativo Común y Régimen Jurídico Básico del Sector Público, Tomo II, Valencia: Tirant Lo Blanch.

GAMERO CASADO, E. y FERNÁNDEZ RAMOS, S. (2017): Manual básico de Derecho Administrativo, Madrid: Tecnos.

GARCÍA DE ENTERRÍA, E. y FERNÁNDEZ RODRÍGUEZ, T. R. (2013): Curso de Derecho Administrativo I, Cizur Menor (Navarra): Thomson Reuters Civitas. 
HEREDIA HERRERA, A. (2007): ¿Qué es un archivo?, Asturias: Trea.

MARTÍN DELGADO, I. (2017): "Ejecutividad y eficacia de los actos administrativos. Las notificaciones administrativas", en GAMERO CASADO, E. (dir.): Tratado de procedimiento administrativo común y régimen jurídico básico del sector público, Valencia: Tirant Lo Blanch.

SANZ LARRUGA, F. J. (2017): "Gestión documental", en GAMERO CASADO, E. (dir.): Tratado de procedimiento administrativo común y régimen jurídico básico del sector público, Valencia: Tirant Lo Blanch.

SENDÍN GARCÍA, M. A. (2009): Régimen jurídico de los documentos. Aspectos administrativos, civiles, penales y procesales, Granada: Comares.

VALCÁRCEL FERNÁNDEZ, P. (2011): “Documentos y archivos electrónicos”, en PIÑAR MAÑAS, J. L. (dir.): Administración electrónica y ciudadanos, 1 . $^{\mathrm{a}}$ ed., Cizur Menor (Navarra): Thomson Reuters Civitas. 